
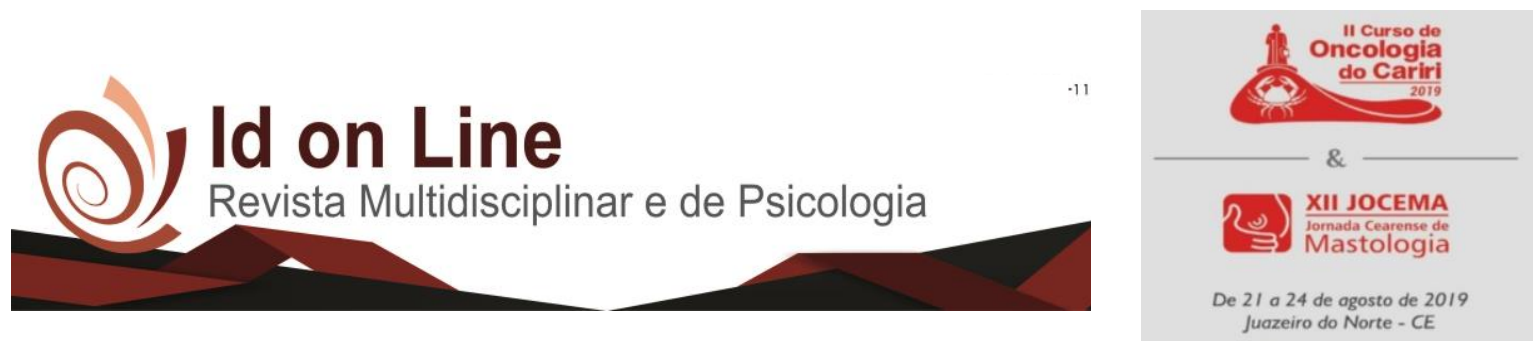

DOI: 10.14295/idonline.v13i46.2014

Resumo

\title{
RASTREIO DE NEOPLASIAS DE PRÓSTATA, PRECAUÇÕES NA SOLICITAÇÃO DO PSA EM PACIENTES IDOSOS: UMA REVISÃO SISTEMÁTICA
}

\begin{abstract}
SOARES, Laryza Souza ${ }^{1}$; MENDES, Isabelle Lima ${ }^{2}$ :MENDES, Ana Carla da Silva ${ }^{3}$; SILVA, Antônio Guilherme Túlio ${ }^{3}$;MORAIS, Letícia Bezerra ${ }^{3}$; PINHO, Jonas lima ${ }^{3}$.
\end{abstract}

Introdução: O Câncer de próstata (CAP) tem média de incidência aos 66 anos. Geralmente, evolui com um bom prognóstico, com expectativa de sobrevida de cerca de 80\%. Entre 2007 a 2018, internações por causa primária de CAP aumentaram gradativamente, com 25287 óbitos registrados, tendo maior prevalência a faixa etária de 70-79 anos. A triagem é feita através do antígeno prostático específico (PSA) com ou sem toque retal no intervalo de 2 a 4 anos para PSA $<1 \mathrm{ng} / \mathrm{ml}$ e entre 1 e 2 anos se $>1 \mathrm{ng} / \mathrm{ml}$. Há praticamente aceitação universal de custo-benefício na triagem para paciente entre 55-69 anos, já para aqueles acima de 70 anos, as recomendações são heterogêneas. Objetivo: Compreender as limitações da solicitação do PSA em pacientes idosos. Metodologia: Trata-se de uma revisão sistemática baseada no protocolo PRISMA, primeiro elaborou-se a pergunta-guia através do anagrama PICOS, definiu-se uma população de estudo, intervenção, conflito e desfecho, resumindo-se em: "Até quando se deve solicitar o PSA em pacientes idosos?" A pesquisa foi feita em inglês com os descritores prostate-specific antigen e prostate e aged nas bases pubmed(936) e scielo(26) sendo que, dos 956, foram selecionados 15, datando de 2012 a 2019. Os critérios de inclusão foram: conter pelo menos dois descritores e responder a pergunta-guia no resumo. Foram excluídos artigos duplicados e com alto teor de viés. Resultados: Um estudo randomizado demonstrou que o rastreio com PSA é custo-efetivo em pacientes entre 55 e 60 anos com intervalos de um ou dois anos e menos custo-efetivo nas idades mais avançadas, reduzindo a sugestão de parada de rastreio entre 59 e 61 anos, antes, essa idade era de 70 a 71 anos. Outros sugerem que se deve individualizar o tempo de triagem entre 55 e 69 anos. Para reduzir gastos em saúde, o médico deve saber identificar quem se beneficiará do rastreio com PSA, já que, aos 65 anos, homens com baixo nível de PSA têm menor risco de diagnóstico de CAP na próxima década, sugerindo abordagem com menos intensidade. O rastreio pode-se iniciar aos 50 anos, ou aos 45 , caso o paciente seja negro ou tenha histórico de CAP na família. Já aos 75 anos, o rastreio deve ser realizado apenas àqueles que tenham expectativa de vida maior que 10 anos. Conclusão: É necessário que haja uma decisão compartilhada entre médico e paciente idoso, para que ocorra uma conduta individualizada, avaliando prognóstico e funcionalidade do paciente, a fim de evitar iatrogenias e ofertar qualidade de vida.

Palavras-chave: antígeno prostático-específico; próstata; idoso.

\footnotetext{
${ }^{1}$ Autora , Acadêmica do curso de medicina na Faculdade de Medicina Estácio de Juazeiro do Norte. E-mail: laryzasouza@gmail.com;

${ }^{2}$ Orientadora, docente na Faculdade de Medicina Estácio de Juazeiro do Norte. E-mail: isabellelimamendes@hotmail.com;

${ }^{3}$ Coautores, Acadêmicos do curso de medicina.
} 


\section{Referências}

ARAFA,M.A. et al. Prostate cancer screening in a low prevalence population- Is it worth it?. Saudi Med Journal. 2017. Vol. 38. N.7.

ARAUJO,F.A.G.R.; SUMITA,N.M; JUNIOR,U.O.B. A continuous fall of PSA use for prostate cancer screening among Brazilian doctors since 2001. Good or bad notice?. Int. braz j urol. (IBJU).Rio de Janeiro. 2019. V. 45. n.3. p.478-485.

AVERY,K.N.L et al. Predictors of Attendance for Prostate-Specific Antigen Screening Tests and Prostate Biopsy. EUROPEAN UROLOGY. 2012. V. 6 2. p. 649 - 655.

CARTER H. B. et al. J Natl Cancer .1999. V. 91. N. 20. P.1733-1737.

GONCALVES-SILVA, A. C. et al. Avaliando as práticas de triagem entre profissionais de saúde em um hospital terciário em São Paulo, Brasil. Clínicas, São Paulo, v. 65, n. 2, p. 151-155, 2010.

HEIJNSDIJK ,E.A.M. et al. J Natl Cancer Inst. 2015. V. 107

INSTITUTO NACIONAL DO CÂNCER. Estimativa 2018: Incidência de Câncer no Brasil. Ministério da Saúde. Rio de Janeiro, 2018. Disponível em < http://www1.inca.gov.br/estimativa/2018/ >. Acesso em 04 de agosto de 2019.

Rastreamento de Câncer de Próstata. Brasil. Nov, 2013. Disponível em < https://www.inca.gov.br/publicacoes/notas-tecnicas/rastreamento-do-cancer-de-prostata >. Acesso em 04 de agosto de 2019.

KLOTZ,L. Active surveillance for favorable-risk prostate cancer: who, how and why? Nature Clinical Practice ONCOLOGY. 2007. Vol. 4,N. 12.

NEAL, D.E. et al. Unanswered questions in screening for prostate cancer. European Journal of Cancer . 2000. V.36, p. 1316-1321.

RIBEIRO, G.M.; Pavan,A.J. Revisão Literária Da Incidência De Casos De Câncer Bucal No Estado Do Paraná. Revista UNINGÁ. 2015. V.24,n.3,p.101-105.

SOCIEDADE BRASILEIRA DE UROLOGIA. Nota oficial SBU e SBPC/ML - Rastreio de Câncer de Próstata. 2018. Disponível em < https://portaldaurologia.org.br/medicos/noticias/nota-oficial-sbue-sbpc-ml-rastreio-de-cancer-de-prostata/ >. Acesso em 04 de agosto de 2019.

TIKKINEN,K.A.O. et al. Prostate cancer screening with prostate-specific antigen (PSA) test: a clinical practice guideline. The BMJ. 2018.

UM TAWFIK. Antígeno Específico da Próstata (PSA) - Triagem Populacional Baseada em Câncer de Próstata: Uma Análise Econômica. Ont Health Techno.l 2015. V.15. N.11. P.1-37.

YAO-LU, G.; STUKEL,T.A; YAO,S.L Prostate-Specific Antigen Screening in Elderly Men. Journal of the National Cancer Institute. 2003. V. 95, N. 23. 